\title{
Lectotipificaciones en Mimosa (Leguminosae) del sur de SUDAMÉRICA
}

\author{
MATÍAS MORALES ${ }^{1,2,3, *}$ y FRANCO CALDERÓN ${ }^{4}$
}

\begin{abstract}
Resumen: Se procedió a realizar la lectotipificación de tres especímenes del género Mimosa L. que crecen en Argentina: M. obstrigosa Burkart, M. tandilensis Speg. y M. callosa var. longiracemosa Burkart. Se describe el procedimiento para la elección de los lectotipos.
\end{abstract}

Palabras clave: Lectotipificación, Leguminosae, Mimosa

Summary: Lectotypifications in Mimosa (Leguminosae) from southern South America. Three taxa of the genus Mimosa (Leguminosae) occurring in Argentina were lectotypified: $M$. obstrigosa Burkart, $M$. tandilensis Speg., and M. callosa var. longiracemosa Burkart. The methodology applied to designate the lectotypes is also described.

Key words: Lectotypification, Leguminosae, Mimosa.

\section{INTRODUCCIÓN}

Se proponen lectotipificaciones de tres especímenes en taxones del género Mimosa L. que crecen en la región nordeste de Argentina, descriptos originalmente para la flora de dicho país pero que crecen además en las áreas adyacentes de Paraguay, Uruguay y Sur de Brasil (Spegazzini, 1901; Burkart, 1946, 1948).

Este trabajo se enmarca en la revisión del género Mimosa que se está desarrollando actualmente para Argentina (M. Morales, R. H. Fortunato y colaboradores, en preparación). Nuestro objetivo

\footnotetext{
${ }^{1}$ Instituto de Recursos Biológicos (CIRN-CNIA, INTA). N. Repetto \& Los Reseros s.n., Hurlingham (1686), Prov. Bs. As., Argentina. E-mails: morales.matias@inta.gob. ar, mmorales0007@gmail.com.

2 Consejo Nacional de Investigaciones Científicas y Técnicas (CONICET), Ciudad de Buenos Aires, Argentina.

3 Facultad de Agronomía y Ciencias Agroalimentarias, Universidad de Morón, Argentina.

${ }^{4}$ Facultad de Ciencias Exactas y Naturales, Universidad Nacional de La Pampa. E-mail: calderonnfran@gmail. com.

${ }^{*}$ Autor en correspondencia.
}

fue lectotipificar especímenes en grupos de especies del género que poseen alta variabilidad morfológica y algunos problemas para su circunscripción mejorando la interpretación de la identidad de los taxones específicos e infraespecíficos.

\section{Materiales y Métodos}

Se examinaron especímenes de diferentes herbarios argentinos y extranjeros visitados: BA, BAB, BAF, CTES, ICN, LP, MBM, MO, MVFA, NY, RB, SI, SP, SPF. Se corroboró la identificación taxonómica de los mismos y se contrastaron los datos de etiqueta con las citas de especímenes tipo en los protólogos de las especies. En el caso de herbarios que no fueron visitados, se los consultó a través de sus portales públicos de internet $(\mathrm{HG}, \mathrm{SF})$.

La lectotipificación se realizó siguiendo las indicaciones del Código Internacional de Nomenclatura para Algas, Hongos y Plantas (McNeill et al., 2012) con las recomendaciones posteriores de McNeill (2014). Para la elección de los lectotipos, se tuvieron en cuenta como criterios prioritarios: 1) la representatividad del espécimen con respecto al protólogo; 2) el lugar de trabajo 
del autor que describió la especie; 3 ) el número de duplicados que presentó o que se localizó en cada sintipo. En caso de no existir material descripto en la publicación original, se determinaron los especímenes que constituyen el material original de la descripción según las indicaciones del Código Internacional de Nomenclatura para Algas, Hongos y Plantas (McNeill et al., 2012), aplicando luego los criterios previamente mencionados.

\section{Tratamiento taxonómico}

1. Mimosa obstrigosa Burkart. Darwiniana 7(2): 231. 1946. Tipo: Argentina. Misiones, Apóstoles, 29-I-1943, Burkart 14291 (Lectotypus SI! aquí designado; isolectotypus HG00064673 [foto!], SF58440 [foto!]). Fig. 1.

Burkart (1946), en la diagnosis traducida al español, menciona las características que diferencian a esta especie: "se diferencia de M. ramulosa Benth. por el porte elevado, las ramas no flexuosas y menos aculeadas, hojas con más folíolos, los capítulos de mayor diámetro y probablemente el lomento más breve...de $M$. sparsa...por el pecíolo brevísimo, las pinnas con más folíolos y más largas, la vaina setosa en todas las partes (no glabra en las caras) y con menos artejos (1-6, no 6-8). Finalmente, de M. sprengelii DC. Difiere por el mayor número de folíolos, los capítulos pedunculados y el fruto que, a más de ser generalmente más corto, lleva cerdas aplicadas al periocarpio, no levantadas como aquella”.

En su publicación original, Burkart (1946), describe esta especie basándose en varios ejemplares y menciona como sintipos (cotipos sensu Burkart, 1946) a los ejemplares: Rodríguez 520, en flor, y Burkart 14291, en fruto. En este trabajo, consideramos que debido a que la diagnosis de $M$. obstrigosa se enfoca principalmente en caracteres vegetativos y carpológicos ("lomentos densamente estrigosos") el espécimen a designar como lectotipo es el que está en fructificación (Burkart 14291). Asimismo, Burkart menciona otros caracteres diagnósticos de $M$. obstrigosa exclusivos de los frutos, como por ejemplo su tamaño, la presencia de pubescencia adpresa sobre los artejos, la densidad de las setas sobre los mismos y el número de artejos.
El ejemplar en fructificación (Burkart 14291, Fig. 1) se ajusta a la descripción original de $M$. obstrigosa por presentar ramas más o menos rectas, con acúleos internodales dispersos, hojas con pecíolos 1-2 $\mathrm{mm}$ de largo, pinnas 1-yugas, cada una de ellas con 16-25 pares de foliólulos, pedúnculos de los capítulos 14-16 $\mathrm{mm}$ de largo, y fruto de 9-11 $\times 4 \mathrm{~mm}$, dehiscencia como un craspedio típico con setas estrigosas, aplicadas, basalmente dilatadas con 2-3 artejos.

2. Mimosa tandilensis Speg. Contribución al estudio de la flora de Tandil: 13. 1901. Tipo: Argentina. Buenos Aires, Tandil, I.1901, Spegazzini s.n. (Lectotypus BAB3155A! aquí designado; isolectotypus BAB3155B!, LPS12568!). Fig. 2.

Spegazzini (1901: 13) cita en el protólogo de la especie que "es muy semejante á la $M$. adpressa Hook. \& Arn. (...) y á la M. sprengellii (...) de las cuales sin embargo se distingue bien por sus cabezuelas de flores pedunculadas, por sus hojas ciliadas en el borde y por las cerdas blanquecinas". Asimismo, luego especifica el área de distribución de la especie: "esta planta es común en todos los cerros, especialmente a lo largo de los arroyos". Sin embargo, no cita ningún espécimen en particular en la publicación original.

A partir de la revisión bibliográfica y visitas a diferentes herbarios, hemos hallado especímenes depositados en LP y BAB coleccionados por C. Spegazzini y E. Aguirre que corresponderían al material original usado para describir $M$. tandilensis. Nos hemos basado en la fecha de recolección de los especímenes y en los datos suministrados por los trabajos de Burkart (1948) y posteriormente de Gutiérrez et al. (2002).

El espécimen E. Aguirre s.n. (LP ex LPS 663) fue recolectado en floración en Sierra de CuraMalal en 1901, y fue mencionado por Burkart (1948) como uno de los sintipos de la especie. El mismo no puede ser considerado lectotipo debido a que la localidad de recolección no es coincidente con el protólogo.

En cuanto a los materiales coleccionados por C. Spegazzini, Burkart (1948) citó varios especímenes depositados en el herbario personal del autor de la especie (LPS, actualmente incorporado al herbario LP) y en el herbario BAB, considerándolos sintipos: Spegazzini s.n. (BAB3155) y Spegazzini (LPS 664). 


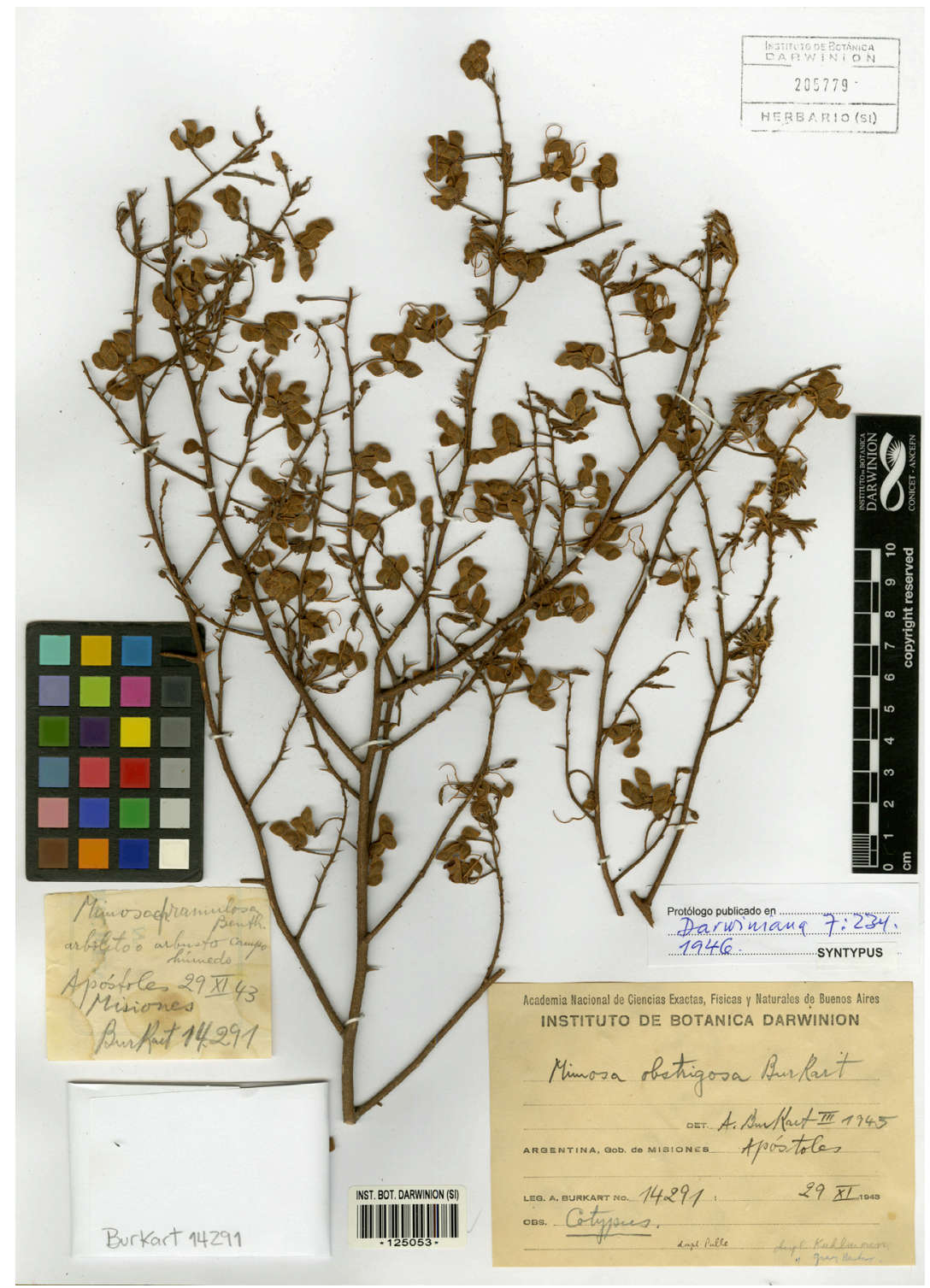

Fig. 1. Lectotipo de M. obstrigosa. Espécimen A. Burkart 14291 (SI).

Gutiérrez et al. (2002) no citaron los especímenes que había mencionado Burkart (1948), sino que hallaron en herbario y consideraron como holotipo de la especie a "Spegazzini 3155 (LP 12568)", basándose en que posee una diagnosis manuscrita. Sin embargo, esto no sería correcto; al describir $M$. tandilensis, el Dr. Spegazzini no se habría basado únicamente en el espécimen LPS12568, debido a que: 1) éste consiste en una rama estéril, y usando exclusivamente este material no habría podido realizar la diagnosis ni completar la descripción (por ejemplo, carece de pedúnculos, carácter citado en el protólogo); 2) dicho espécimen presenta duplicados en $\mathrm{BAB}$, donde el autor depositaba también sus materiales; 3 ) existe otro espécimen, Aguirre s.n. (LP) que formaría parte del material original estudiado por el autor.

La designación del espécimen Spegazzini 3155 (LP S12568) como holotipo (Gutiérrez et al., 2002) no sería adecuada, ya que como se infiere del Código 
Bol. Soc. Argent. Bot. 53 (1) 2018

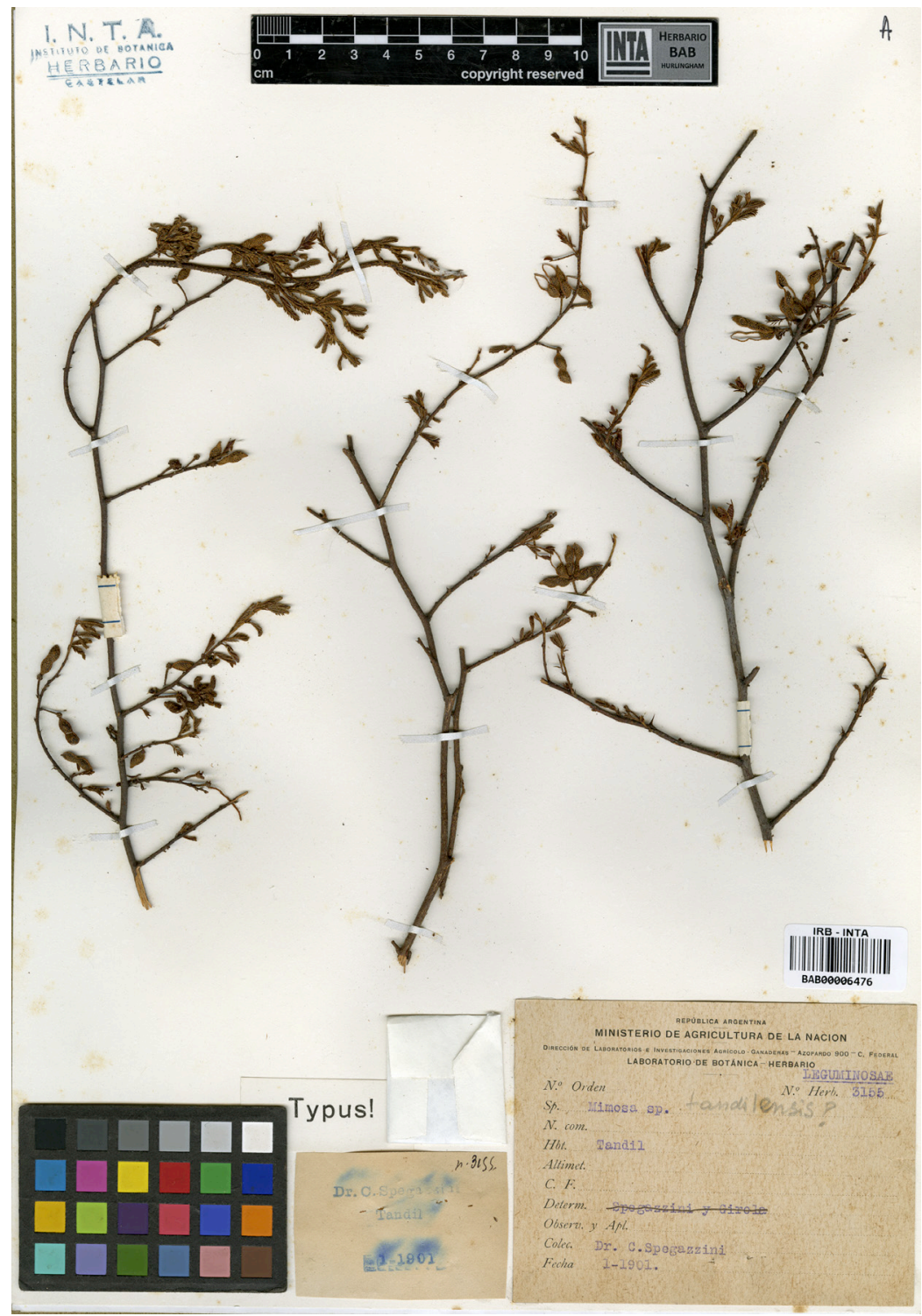

Fig. 2. Lectotipo de M. tandilensis. Espécimen Spegazzini s.n. (BAB 3155A).

Internacional de Nomenclatura para Algas, Hongos y Plantas (McNeill et al., 2012) en su artículo 9.11, cuando no se indica específicamente el material tipo en la publicación original, sólo debe considerarse que hay un holotipo si exclusivamente se ha analizado un solo espécimen. Sólo podría asegurarse que hay holotipo si se indica particularmente un herbario en el protólogo (y si únicamente un duplicado del espécimen fue depositado allí) o si se aclaró en la publicación (o hay evidencia firme) que únicamente dicho espécimen fue utilizado para la descripción (McNeill et al., 2012; McNeill, 2014). Por lo expuesto anteriormente, en el caso de M. tandilensis existen suficientes indicios de que estos supuestos se incumplen. Si bien la designación de Gutiérrez et al. (2002) podría catalogarse como una "lectotipificación inadvertida", no puede considerarse como tal porque se realizó posterior a 2001 (Prado et al., 2015). Por estas razones, no existe un holotipo de $M$. tandilensis, sino varios sintipos. 
Burkart (1948) también ha mencionado especímenes de BAB (C. Spegazzini BAB3155) entre el material original de M. tandilensis. En este herbario, hemos hallado dos especímenes diferentes con el número 3155, ambos coleccionados en 1901 en Tandil. Revisando los registros históricos de $\mathrm{BAB}$, pudimos constatar que " 3155 " es un número del herbario (no de colector) y por ende ambos especímenes deben ser considerados BAB3155A y BAB3155B. El espécimen BAB3155A (Fig. 2) posee frutos y restos de flores y se encuentra en mejor estado de preservación que $\mathrm{BAB} 3155 \mathrm{~B}$, por lo que reproduce más fielmente la diagnosis y descripción originales, además de contener la localidad del protólogo. Por lo tanto, se designa a este espécimen como el lectotipo de la especie.

Como una observación adicional, en el herbario LP donde se incorporaron los materiales del herbario personal del Dr. Spegazzini, no hemos encontrado el material citado por Burkart (1948) con el número LPS664. Este material consistía en ramas no montadas que no se asemejan al espécimen de Spegazzini (LP 12568) citado por Gutiérrez et al. (2002). Es posible que ambos especímenes fueran diferentes.

Esto se ve reforzado por el hecho que LP12568 posee el número " 3155 " agregado en una etiqueta aparte, que caligráficamente coincide con el número 3155 de los especímenes y del registro de BAB. De todo esto se deduce que posiblemente LP12568 haya sido un duplicado enviado desde BAB y que el espécimen LPS664 haya desaparecido o no haya podido ser encontrado exitosamente hasta la actualidad. No obstante, ambos especímenes (LP12568 y LPS664) deben ser considerados en el material original estudiado por el Dr. Spegazzini.

\section{Mimosa longiracemosa (Burkart) Barneby}

$\equiv$ Mimosa callosa Benth. var. longiracemosa Burkart. Darwiniana 8: 192. Tipo: Argentina. Misiones, Santa Ana, en campos, 18.IX.1912, F. M. Rodríguez 626 (Lectotypus SI2229! aquí designado; isolectotypus: BA51279A!, BA51279B!, F, LIL, NY!, SIs.n.!, 2228!, 2230!, 2232!). Fig. 3.

Burkart (1948) describe por primera vez a M. callosa var. longiracemosa, que actualmente se considera con rango específico (Barneby, 1991). En su diagnosis, diferencia esta variedad de la variedad típica por caracteres mayormente detectables en los especímenes floríferos: "esta variedad se distingue de la especie por sus racimos muy largos y poblados con gran número (15-27) de capítulos; el ápice del eje principal del racimo no termina en capítulo grande que florece y fructifica antes que los laterales, sino que su floración y fructificación progresa -como en un racimo típico- de abajo hacia el ápice, de modo que los capítulos fructíferos se encuentran en la base o el medio del racimo y no en su ápice; brácteas de las flores superiores glabras y eciliadas, con margen finamente glanduloso, de ápice muy incurvo" (Burkart, 1948: 192-193).

Burkart (1948) designó dos especímenes como tipos nomenclaturales de $M$. callosa var. longiracemosa, uno en flor y otro en fruto, ambos correspondientes a $F$. M. Rodríguez 626 pero en distintas cartulinas, por lo que ambos son sintipos de dicho taxón. En el Instituto de Botánica Darwinion, lugar de trabajo del Dr. Burkart, hemos hallado varios especímenes con diferente numeración de herbario, algunos en flor y otros en fruto.

El espécimen Rodríguez 626 (SI2229) se encuentra en floración y, por lo tanto, exhibe con mayor fidelidad la diagnosis de la publicación original, con respecto a los especímenes en fructificación y a otros con floración fragmentada (Fig. 3). Siendo este el criterio prioritario se considera lectotipo.

Asimismo, el espécimen SI2229 posee una anotación manuscrita del autor del taxón, con parte de la descripción. En consecuencia, de acuerdo con los criterios prioritarios y recomendaciones establecidos en el Código Internacional de Nomenclatura para Algas, Hongos y Plantas (McNeill et al., 2012), se designa a este espécimen como lectotipo.

\section{Agradecimientos}

Agradecemos a los curadores de todos herbarios visitados por tener acceso al material revisado, en especial al personal de los herbarios BAB, LP y SI. Asimismo, a María Monsalvo y Manuel Belgrano por el escaneo de los especímenes tipo. Destacamos 
Bol. Soc. Argent. Bot. 53 (1) 2018

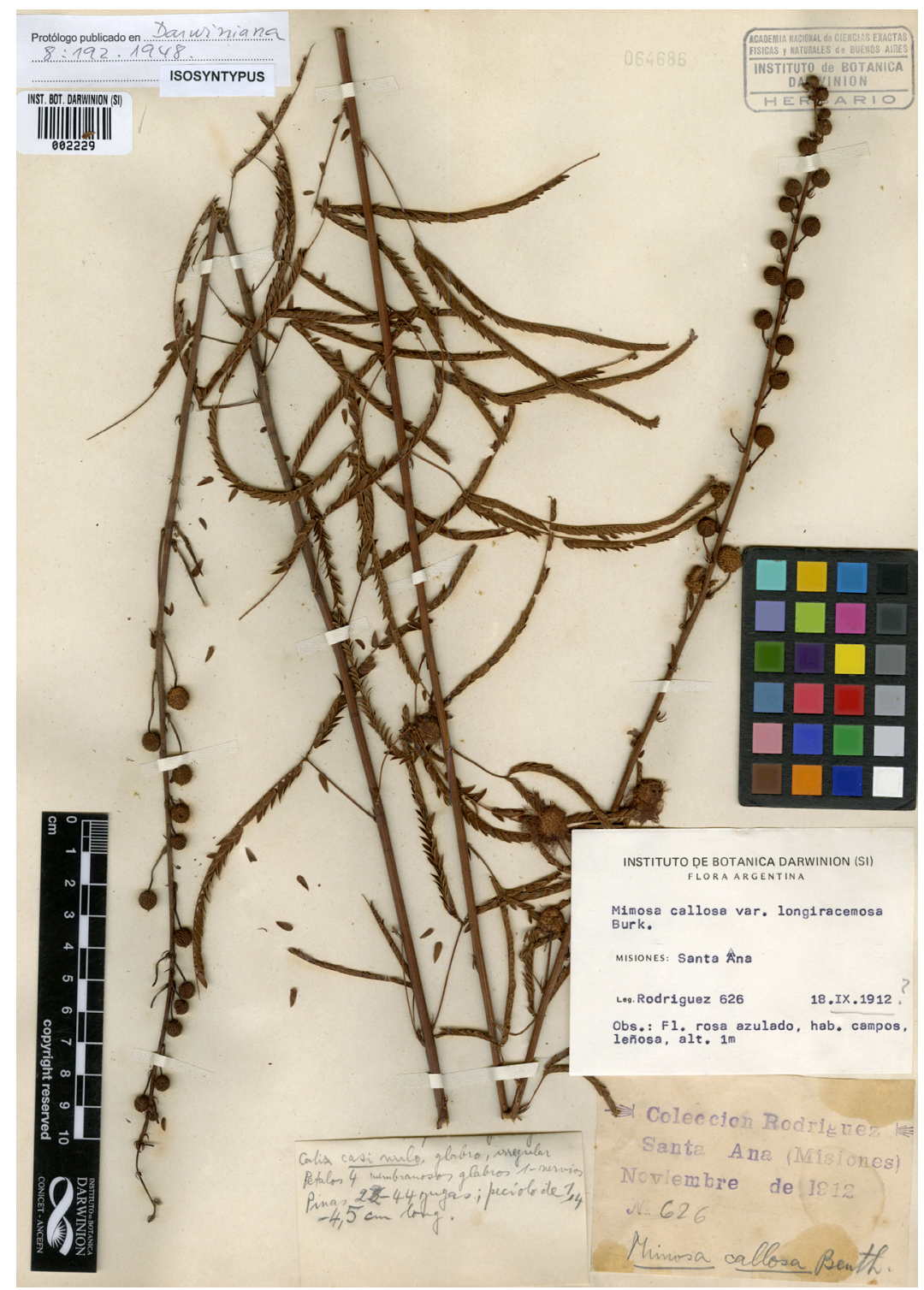

Fig. 3. Lectotipo de M. callosa var. longiracemosa. Espécimen Rodríguez 626 (SI 2229).

la valiosa colaboración y orientación de la Dra. Renée H. Fortunato. Las siguientes instituciones financiaron viajes y visitas a herbarios: CONICET (PIP112-20110100250, y Financiamiento Parcial de Estadías en el Exterior para Becas Post-doctorales año 2012), Agencia Nacional de Promoción Científica y Tecnológica (PICT 2011-821), PID 06002-16) y Troels Myndel Foundation (2009, visita a herbarios de Brasil).

\section{Bibliografía}

BARNEBY, R. C. 1991. Sensitivae Censitae: Mimosa. Mem. New York Bot. Gard. 65: 1-835.

BURKART, A. 1946. Leguminosas nuevas o críticas. Darwiniana 7: 216-239.

BURKART, A. 1948. Las especies argentinas del género Mimosa. Darwiniana 8: 9-231.

GUTIÉRREZ, D., L. KATINAS \& S. S. TORRES ROBLES. 2002. Type material of Carlos L. 
Spegazzini in the Museo de la Plata Herbarium (LP), Argentina. II: Fabaceae. Darwiniana 40: 77-101.

MCNEILL, J. 2014. Holotype specimens and type citations: General issues. Taxon 63: 112-113.

MCNEILL, J., F. R. BARRIE, W. R. BUCK, V. DEMOULIN, W. GREUTER, D. L. HAWKSWORTH, P. S. HERENDEEN, S. KNAPP, K. MARHOLD, J. PRADO, W. F. PRUD'HOMME VAN REINE, G. F. SMITH, J. H. WIERSEMA \& N. J. TURLAND. 2012. International code of nomenclature for algae, fungi, and plants (Melbourne Code). Adoptado por XVIII International Botanical Congress Melbourne, Australia, Julio 2011. Koeltz Scientific Books, Königstein, 208 pp. [Regnum Vegetabile 154]. Disponible en: http:/www.iapttaxon.org/nomen/. [Acceso: 05 de Abril de 2017].
PRADO, J. F., R. Y. HIRAI \& R. C. MORAN. 2015. (046-048) Proposals concerning inadvertent lectotypifications (and neotypifications). Taxon 64: 651 .

SPEGAZZINI, C. L. 1901. Contribución al estudio de la Flora de Tandil. Sesé, Larrañaga y Renovales, La Plata-Buenos Aires.

Recibido el 3 de mayo de 2017, aceptado el 28 de julio de 2017. Editor: Franco E. Chiarini. 
